\title{
Chemical and microbiological stability study of a diazepam suspension
}

J Rocha', T Ribeiro', P Correia'

1 Escola Superior de Saúde, Instituto Politécnico do Porto, Portugal

\section{Introduction:}

Drugs produced by pharmaceutical industry are limited to some dosages and forms that do not cover all therapy needs. Thus, the compounding drugs appear as a custom solution for several situations. One of those formulations is the diazepam suspension, produced in some hospitals with an empirical expiration date of 14 days.

\section{Objectives:}

To study the microbiological and chemical stability of diazepam suspensions in order to assess its expiration date.

\section{Methods:}

Samples of the suspension of diazepam $0.4 \mathrm{mg} / \mathrm{ml}$ were prepared, from which both experimental studies were carried out. The chemical stability study was performed adapting the dosing method described in the Portuguese Pharmacopeia for tablets of diazepam. The validation of the dosing method included parameters of linearity (calibration curve), precision (repeatability) and accuracy (recovery). The concentrations of the diazepam suspension were determined at day $1,2,3,6,7,8$ and 14 of the study. In the case of the microbiological study, a non-selective culture medium was used and the pour plate method was implemented. Assays were weekly performed, up to 8 weeks after preparation.

Results: 
The analytical method of determination proved to be linear, as $R=0,999$. Moreover, precision and accuracy values were within the defined limits. The results showed that the diazepam content remained stable for 7 days, within the therapeutic dosage. The microbiological study showed no alterations in the suspension until the 39th day, although microbiological growth was observed after the 49th day assay.

\section{Conclusions:}

It was concluded that the given expiration date for diazepam suspensions (14 days) is incompatible with the chemical stability of the drug, although the microbiological stability is more extensive, suggesting a new expiration date of 7 days. However, a more accurate expiration date can only be established if a stability study is carried out with a wide range of samples and different storage conditions. 